\title{
Development of New Solitary Retinoblastoma Tumors during and after Chemotherapy
}

\author{
Won Jong Choi ${ }^{1}$, Dong Hyun Jo ${ }^{2}$, Hyoung Jin $\mathrm{Kang}^{3}$, Hee Young Shin ${ }^{3}$, Young Suk Yu ${ }^{1}$, Jeong Hun Kim, ${ }^{1,4}$ \\ ${ }^{1}$ Department of Ophthalmology, Seoul National University College of Medicine, Seoul, Korea \\ ${ }^{2}$ Department of Anatomy and Cell Biology, Seoul National University College of Medicine, Seoul, Korea \\ ${ }^{3}$ Department of Pediatrics, Cancer Research Institute, Seoul National University College of Medicine, Seoul, Korea \\ ${ }^{4}$ Fight against Angiogenesis-Related Blindness (FARB) Laboratory, Biomedical Research Institute, Seoul National University \\ Hospital, Seoul, Korea \\ ${ }^{5}$ Department of Biomedical Sciences, Seoul National University College of Medicine, Seoul, Korea
}

Purpose: To review the occurrence of new solitary tumors during and after intravenous chemotherapy against retinoblastoma.

Methods: From 115 eyes of 78 patients with a diagnosis of intraocular retinoblastoma who underwent intravenous chemotherapy and focal treatment without prior treatment, patient demographics, age at diagnosis, laterality, classification (Reese-Ellsworth and International Classification of Retinoblastoma), and treatment options were recorded. In addition, the occurrence of small tumors during and after chemotherapy was documented with a detailed review of medical records and fundus photographs.

Results: Of a total of 115 eyes of 78 consecutive patients, new solitary tumors were observed in 50 eyes (50 / 115, 43\%) of 40 patients (40 / 78, 51\%). Multinominal logistic regression analyses showed that age at diagnosis (before 1 year) and vitreal seeding at diagnosis were linked to the development of isolated and miliary tumors, respectively. Kaplan-Meier analyses demonstrated that all small tumors developed with 20 months from the start of chemotherapy. Twenty-eight eyes (28 / 34 , $82 \%)$ were salvaged with additional focal treatment in 34 eyes with isolated tumors.

Conclusions: Small tumors were observed during and after chemotherapy against retinoblastoma in patients who underwent intravenous chemotherapy and focal treatment. It is necessary to promptly identify and address small tumors for the preservation of eyeball and vision.

Key Words: Chemotherapy, Neoplasms, Retina, Retinoblastoma

Recently, most retinoblastoma patients receive eye-preserving treatment as a primary therapeutic option $[1,2]$. For

Received: August 31, 2020 Final revision: November 30, 2020

Accepted: December 9, 2020

Corresponding Author: Jeong Hun Kim, MD, PhD. Fight against Angiogenesis-Related Blindness (FARB) Laboratory, Biomedical Research Institute, Seoul National University Hospital, 101 Daehak-ro, Jongno-gu, Seoul 03080, Korea. Tel: 82-2-740-8387, Fax: 82-2-741-3187, E-mail: steph25@snu.ac.kr patients with unilateral diseases, intra-arterial chemotherapy is employed for the control of initial tumors which cannot be addressed with focal treatment options [3]. On the other hand, for patients with bilateral diseases, intravenous or intra-arterial chemotherapy is the mainstay treatment option except in selected cases with small tumors which can be managed with focal treatment options, mostly thermotherapy [3]. In these clinical settings, it is more important to control newly developed or recurrent retinoblastoma 
tumors during and after chemotherapy in that their management determines the overall rate of eyeball salvage and resultant visual outcomes.

In particular, it is known that new tumors develop in the early phase during and after the treatment of retinoblastoma [4-6]. Therefore, it is possible to address them with intensive and meticulous follow-up examination within the first 2 to 3 years. Another concern is whether there are differential patterns of new tumor development. Although previous works on new tumors well defined its development, our retrospective reviews on consecutive retinoblastoma patients additionally found two distinct patterns of new tumors, isolated and miliary tumors. Further analyses on these development patterns might enhance our understanding on these phenomena and the potential of retinoblastoma control.

To meet these purposes, this study was conducted to investigate the occurrence of new small tumors during and after chemotherapy and determine prognostic factors for the effective management of them.

\section{Materials and Methods}

This study was a single-center, retrospective, observational case series. The study was approved by the institutional review board of Seoul National University Hospital (1601-026-733) to review and collect the clinical data of patients with retinoblastoma who visited Seoul National University Children's Hospital from January 1, 2004 to December 31, 2014. For this type of study formal consent was not required. The review of electronic health records was conducted on March 3, 2020. All study protocols conformed to the tenets of the Declaration of Helsinki.

Patients who were diagnosed with intraocular retinoblastoma and treated by intravenous chemotherapy and focal treatment were included in this study. Exclusion criteria were prior treatment in other institutions, other hereditary retinal diseases, and follow-up loss before the completion of six cycles of chemotherapy. In total, 115 eyes of 78 patients were identified and further analyzed. Demographic findings, age at diagnosis, sex, laterality, treatment options, the start date of the first chemotherapy cycle, the end date of the final chemotherapy cycle, and the date of enucleation were recorded from the electronic health records of Seoul National University Hospital. The classifi-
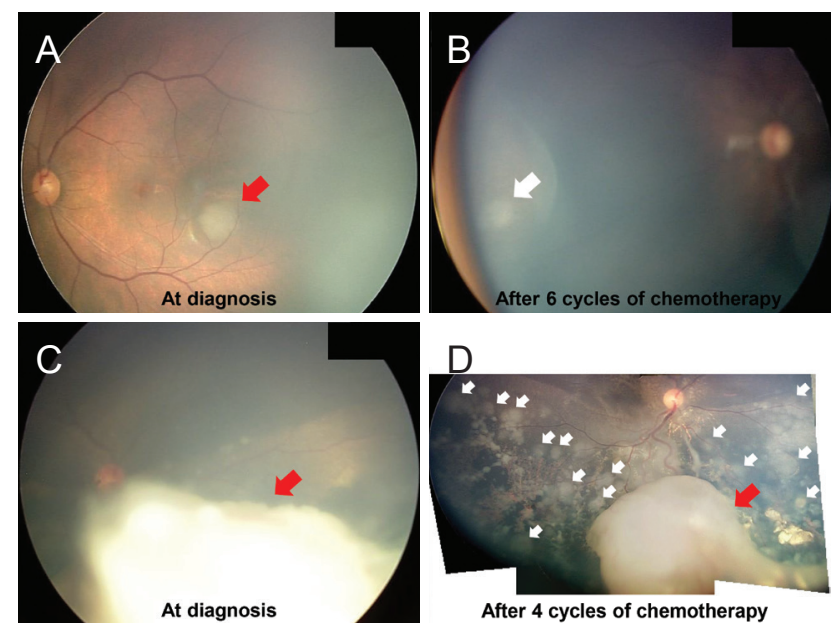

Fig. 1. Representative fundus photographs of patients with new small retinoblastoma tumors during and after chemotherapy. Original tumors and newly developed tumors were indicated with red and white arrows, respectively. $(\mathrm{A}, \mathrm{B})$ Isolated tumors and $(\mathrm{C}, \mathrm{D})$ miliary tumors.

cation of retinoblastoma was determined according to the Reese-Ellsworth classification and International Classification of Retinoblastoma (Murphree version) [7] based on the detailed descriptions of examination from the medical records and fundus photographs. In addition, the occurrence of new small tumors was documented during and after chemotherapy from the initiation of chemotherapy. According to the development patterns, isolated and miliary tumors were identified. Isolated and miliary tumors were defined as solitary tumors (the number was less than 10 at an examination) and clustered tumors of which numbers were more than 10 at an examination, respectively. The representative photographs of isolated and miliary tumors were provided in Fig. 1A-1D.

As previously described, patients received intravenous chemotherapy based on cisplatin or carboplatin depending on the period [1,8]. 1) Regimen 1 (from 2004 to 2007): cisplatin $\left(60 \mathrm{mg} / \mathrm{m}^{2}\right.$ on day 0$)$, etoposide $\left(100 \mathrm{mg} / \mathrm{m}^{2}\right.$ on day 2 and 5), doxorubicin (30 $\mathrm{mg} / \mathrm{m}^{2}$ on day 2), and cyclophosphamide (30 mg/kg on day 3 and 4); 2) Regimen 2 (from 2007 to 2010 ): carboplatin ( $450 \mathrm{mg} / \mathrm{m}^{2}$ on day 0 ), etoposide $\left(100 \mathrm{mg} / \mathrm{m}^{2}\right.$ on day 2 and 5$)$, vincristine $\left(2 \mathrm{mg} / \mathrm{m}^{2}\right.$ on day 0 ), doxorubicin (30 mg/m $\mathrm{m}^{2}$ on day 2 ), and cyclophosphamide ( $30 \mathrm{mg} / \mathrm{kg}$ on day 3 and 4); and 3) Regimen 3 (from 2010 to 2014): carboplatin ( $450 \mathrm{mg} / \mathrm{m}^{2}$ on day 2 ), etoposide $\left(100 \mathrm{mg} / \mathrm{m}^{2}\right.$ on day 0 and 1$)$, vincristine $\left(2 \mathrm{mg} / \mathrm{m}^{2}\right.$ on day 0 ), doxorubicin (30 mg/m $\mathrm{m}^{2}$ on day 0 ), and cyclophospha- 
mide (30 mg/kg on day 1 and 2). Upon the completion of the chemotherapy sessions, patients underwent 3 consecutive examination under anesthesia to confirm the regression status of retinoblastoma. Then, the fundus was examined every 2 to 4 months with examination under anesthesia or at the outpatient clinic (the frequency of examination under anesthesia was 2 to 4 times per year).

Comparison of age at diagnosis and follow-up period was performed using Kruskal-Wallis test and post hoc Dunn's multiple comparison test among patients with isolated tumors, with miliary tumors, and without new small tumors. Pearson's chi-square test was utilized to figure out any difference in the proportion of females among groups. The correlation between two different retinoblastoma classification schemes and the development of isolated or miliary tumors was investigated with Fischer's exact test. The effects of demographic/clinical characteristics (age at diagnosis, sex, and laterality) and tumor characteristics (the existence of subretinal and vitreal seedings at initial diagnosis) on the development of isolated or miliary tumors were analyzed with multinomial logistic regression analyses. Patients were divided into two groups according to whether they were diagnosed with retinoblastoma before the age of 1 year. In this manner, age at diagnosis was analyzed as a discrete variable. Pearson's chi-square test was implemented to figure out the relationship between RB1 gene mutation and the development of new tumors. Kaplan-Meier analyses were utilized to investigate the temporal patterns of the occurrence of newly developed tumors. Treatment outcomes among groups were analyzed using Pearson' chi-square test. All statistical analyses were performed using IBM SPSS Statistics ver. 22.0 (IBM Corp., Armonk, NY, USA).

\section{Results}

In an 11-year study period, 115 eyes of 78 patients were identified to be diagnosed with intraocular retinoblastoma and treated with intravenous chemotherapy and focal treatment. Among them, the number of females was 34 (44\%). The mean age at diagnosis was $14 \pm 13$ months (median, 11 months; range, 1-63 months). Bilateral diseases were identified in 48 patients $(62 \%)$. The mean follow-up period was $96 \pm 45$ months (median, 95 months; range, 6-185 months).
From the 115 eyes of 78 consecutive retinoblastoma patients, new small tumors were observed in 50 eyes (43\%) of 40 patients $(51 \%)$ during and after chemotherapy until 24 months after the initiation of chemotherapy. There was no significant difference among the regimens as the matter of development of solitary tumor, even if we sub-classify the regimens by eyes $(p=0.247)$ and patients $(p=0.698)$. All these cases had a tumor-free-period of less than 1 year and 8 months. Then, the tumors were divided into two distinct categories, isolated and miliary tumors, according to the distribution and occurrence patterns (Fig. 1). Isolated and miliary tumors were identified in 34 eyes $(34 / 50$, $68 \%)$ of 25 patients $(25 / 41,61 \%)$ and 16 eyes $(16 / 50,32 \%)$ of 16 patients $(16 / 41,39 \%)$, respectively. One patient had a new isolated tumor in one eye and new miliary tumors in the other eye.

Clinical characteristics of patients and tumor classification per eye were summarized in Table 1 and 2. There was no significant difference in the proportion of female and follow-up period. In contrast, Reese-Ellsworth classification of retinoblastoma among groups with isolated tumors, with miliary tumors, and without new small tumors was significantly different (Table 2). Moreover, patients with isolated tumors were younger than those with miliary tumors and without new small tumors when they were diagnosed with retinoblastoma (Table 1). However, patients with RB1 gene mutation were not related to the development of new tumor $(p=0.546)$.

A series of multinomial logistic regression analyses were performed to investigate the prognostic factors for the development of new small retinoblastoma tumors during and after chemotherapy. Among clinical and demographic characteristics (age at diagnosis, sex, and laterality), age at diagnosis under 1 year was the only significant prognostic factor for the development of isolated tumors (Table 3). In addition to the tumor grading according to International and Reese-Ellsworth classification schemes, further analyses were performed using subretinal and vitreal seeding as alternative prognostic factors. As in Table 4, vitreal seeding was the significant prognostic factor for the development of miliary tumors.

Kaplan-Meier analyses showed that all small tumors developed before 20 months from the initiation of chemotherapy (Fig. 2A, 2B). As for isolated tumors, the mean number of occurrence was 1 (median, 1; range, 1-4) and that of total tumors was 2 (median, 1; range, 1-12). Upon 
Table 1. Clinical and demographic features of patients with intraocular retinoblastoma

\begin{tabular}{lccc}
\hline Feature & $\begin{array}{c}\text { Patients with new isolated tumors } \\
\text { (25 patients) }\end{array}$ & $\begin{array}{c}\text { Patients without new isolated tumors } \\
\text { (53 patients) }\end{array}$ & $p$-value \\
\hline Age at diagnosis (mon) & $8 \pm 7$ & $17 \pm 15$ & $0.001^{*}$ \\
Sex (male : female) & $15: 10$ & $29: 24$ & $0.661^{\dagger}$ \\
Follow-up period (mon) & $98 \pm 49$ & $95 \pm 43$ & $0.785^{\ddagger}$ \\
\hline
\end{tabular}

*Mann-Whitney $U$-test; ${ }^{*}$ Pearson's chi-square test; ${ }^{\star} t$-test.

Table 2. Classification of retinoblastoma in eyes with intraocular retinoblastoma

\begin{tabular}{|c|c|c|c|c|}
\hline Feature & $\begin{array}{l}\text { Eyes with isolated tumors } \\
\text { (33 eyes) }\end{array}$ & $\begin{array}{l}\text { Eyes with miliary tumors } \\
\text { (16 eyes) }\end{array}$ & $\begin{array}{l}\text { Eyes without new small tumors } \\
\text { (65 eyes) }\end{array}$ & $p$-value \\
\hline Reese-Ellsworth & & & & 0.021 \\
\hline $1 \mathrm{~A}$ & 2 & 0 & 1 & \\
\hline $1 \mathrm{~B}$ & 2 & 0 & 4 & \\
\hline $2 \mathrm{~A}$ & 5 & 0 & 3 & \\
\hline 2B & 8 & 0 & 6 & \\
\hline $3 \mathrm{~A}$ & 1 & 0 & 6 & \\
\hline $3 \mathrm{~B}$ & 5 & 2 & 12 & \\
\hline $4 \mathrm{~A}$ & 10 & 9 & 23 & \\
\hline $4 \mathrm{~B}$ & 0 & 0 & 0 & \\
\hline $5 \mathrm{~A}$ & 0 & 0 & 3 & \\
\hline $5 \mathrm{~B}$ & 0 & 5 & 7 & \\
\hline International Classification & & & & 0.097 \\
\hline A & 3 & 0 & 8 & \\
\hline $\mathrm{B}$ & 12 & 1 & 15 & \\
\hline $\mathrm{C}$ & 6 & 1 & 7 & \\
\hline $\mathrm{D}$ & 13 & 14 & 33 & \\
\hline $\mathrm{E}$ & 0 & 0 & 2 & \\
\hline
\end{tabular}

Table 3. Clinical and demographic characteristics linked to the development of small tumors

\begin{tabular}{lcccc}
\hline Characteristics & $\chi^{2}$ & $p$-value & $\begin{array}{c}\text { Odds ratio for isolated tumors } \\
(95 \% \text { confidence interval })\end{array}$ & $\begin{array}{c}\text { Odds ratio for miliary tumors } \\
(95 \% \text { confidence interval) }\end{array}$ \\
\hline Age at diagnosis $(<1$ yr vs. $>1$ yr) & 9.343 & 0.009 & $4.398(1.174-16.477)$ & $0.431(0.113-1.650)$ \\
Sex (female vs. male) & 1.691 & 0.429 & $0.747(0.230-2.423)$ & $1.983(0.551-7.146)$ \\
Laterality (unilateral vs. bilateral) & 3.329 & 0.189 & $0.407(0.105-1.579)$ & $1.723(0.486-6.109)$ \\
\hline
\end{tabular}

the identification of new small tumors, various treatment modalities were employed to address them as soon as possible. For isolated tumors (34 eyes), focal treatment options were utilized, including thermotherapy (27 eyes, 79\%), thermotherapy and cryotherapy (three eyes, 9\%), laser photocoagulation (two eyes, 6\%), laser photocoagulation and thermotherapy (one eye, 3\%), and thermotherapy and proton beam irradiation (one eye, $3 \%$ ), resulting in the sal- 
Table 4. Tumor characteristics linked to the development of small tumors

\begin{tabular}{lrccc}
\hline Characteristics & $\chi^{2}$ & $p$-value & $\begin{array}{c}\text { Odds ratio for isolated tumors } \\
(95 \% \text { confidence interval })\end{array}$ & $\begin{array}{c}\text { Odds ratio for miliary tumors } \\
(95 \% \text { confidence interval })\end{array}$ \\
\hline Subretinal seeding & 8.173 & 0.094 & $0.778(0.327-1.853)$ & $5.769(0.688-48.360)$ \\
Vitreal seeding & 12.701 & 0.002 & $0.000(0.000-0.000)$ & $5.333(1.367-20.803)$ \\
\hline
\end{tabular}

Table 5. Outcomes according to the development of new small tumors

\begin{tabular}{lcccc}
\hline Outcomes & $\begin{array}{c}\text { Eyes with isolated tumors } \\
\text { (34 eyes) }\end{array}$ & $\begin{array}{c}\text { Eyes with miliary tumors } \\
\text { (15 eyes) }\end{array}$ & $\begin{array}{c}\text { Eyes without new small tumors } \\
\text { (65 eyes) }\end{array}$ & $p$-value \\
Enucleation & 6 & 13 & 10 & $<0.001$ \\
Salvage & 28 & 2 & 55 \\
\hline
\end{tabular}

A

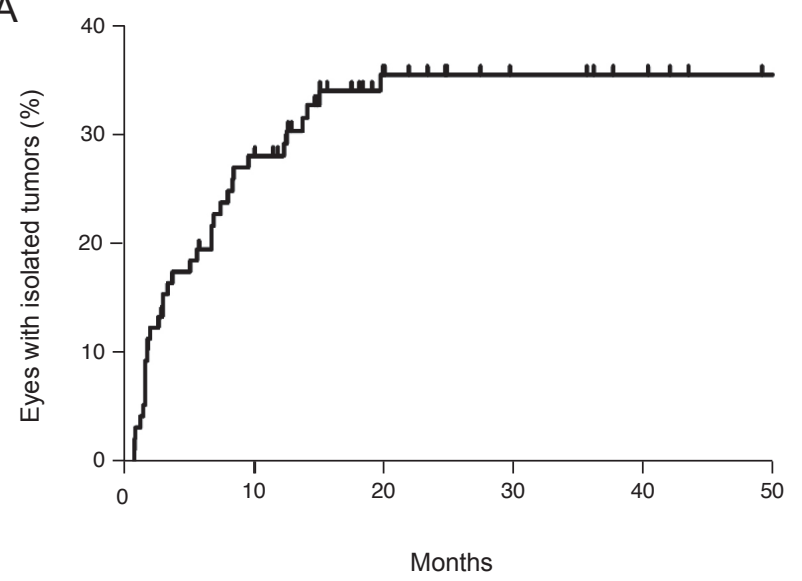

B

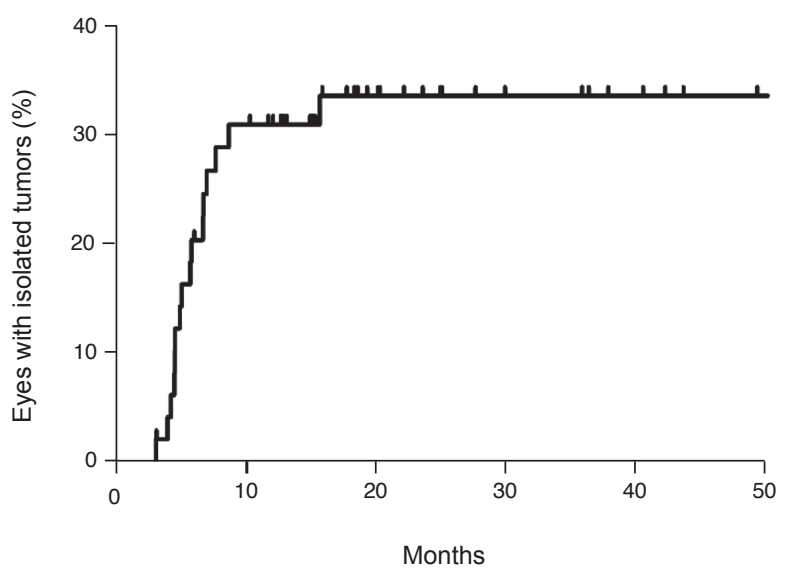

Fig. 2. Kaplan-Meier estimates of eyes with (A) isolated and (B) miliary tumors during and after chemotherapy against retinoblastoma.

vage of 28 eyes $(82 \%)$. On the other hand, for miliary tumors (16 eyes), eight eyes (50\%) were enucleated without further treatment, five eyes $(31 \%)$ were enucleated despite laser photocoagulation, thermotherapy and cryotherapy, proton beam irradiation, and thermotherapy. The other two cases (13\%) were treated with thermotherapy (one eye) and intra-arterial chemotherapy and thermotherapy (one eye), resulting in the salvage of eyeballs. One patient did not come back to the hospital (Table 5).

\section{Discussion}

This study is an observational case series to investigate the occurrence of new small tumors during and after intravenous chemotherapy and focal treatment for retinoblasto- ma. From 115 eyes of 78 retinoblastoma patients who underwent intravenous chemotherapy in 11-year study period, new small tumors were observed in 50 eyes $(43 \%)$ of 40 patients (51\%) during and after chemotherapy. Isolated and miliary tumors were identified in 34 eyes ( $34 / 50,68 \%)$ of 25 patients $(25 / 40,63 \%)$ and 16 eyes $(16 / 50,32 \%)$ of 16 patients $(16 / 40,40 \%)$, respectively. One patient had a new isolated tumor in one eye and a new miliary tumor in the other eye. The proportion of cases of new small tumors was comparable to that in a previous study of 50 eyes of 32 hereditary retinoblastoma patients $(48 \%$ of the treated eyes) [5].

During the fundus examination during and after chemotherapy, two distinct patterns of small tumors were identified, isolated and miliary tumors. They were defined according to the occurrence and distribution patterns of 
small tumors. Isolated tumors developed in the more peripheral area than the original tumors. The median numbers of occurrence and total tumors were 1, demonstrating that isolated tumors usually occur once during the follow-up period. In contrast, miliary tumors often formed clusters, involve 2 or more quarters of the retina, and were innumerable by gross observation. All these tumors were apart from original tumors, including subretinal or vitreous seeding, evidencing their de novo formation.

Previous studies demonstrated that most cases of new tumors were detected within a 1-year follow-up period after the completion of chemotherapy [5,6], as all cases were calculated to occur within 20 months from the initiation of chemotherapy with Kaplan-Meier analyses in this study. In a study with 50 eyes of 32 patients with hereditary retinoblastoma, Schueler et al. [5] also showed that most de novo tumors occurred within 7 months after the completion of chemotherapy. In another study with 162 eyes of 106 retinoblastoma patients, Shields et al. [6] demonstrated that new tumor development occurred in $23 \%$ of patients at 1 -year follow-up, followed by $1 \%$ increase at 5 -year follow-up. Similarly, most cases with new retinoblastoma tumors were found within 26 months after initiation of external beam radiation using a linear accelerator [4].

From multinomial logistic regression analyses using clinical (age at diagnosis, sex, and laterality) and tumor (subretinal and vitreal seeding) characteristics as prognostic factors, age at diagnosis $(<1$ year) and vitreal seeding were found to be linked with the development of isolated and miliary tumors, respectively. Age at diagnosis is also identified to be the risk factor for de novo tumor development in a previous study with hereditary retinoblastoma patients [5]. The spatiotemporal patterns of retinal development, from central to periphery, might be the reason [9]. When the retina was divided into macula, macula-equator, and equator-ora, all small tumors were identified in the same area or more peripheral area than original tumors in this study. It is also remarkable that vitreal seeding was the prognostic factor for the development of miliary tumors and all the small tumors in the form of miliary tumors were apart from the original tumor. Currently, molecular mechanisms of new small retinoblastoma tumors are yet to be found. However, soluble factors from the original tumors might be a causative factor of miliary nature of new small tumors.

In line with previous studies of isolated tumors, $82 \%$ of eyes with isolated tumors were effectively treated with focal treatment options. Although specific focal treatment options varied depending on the period, there was no definite difference among different treatment options in the treatment outcomes. Unlike isolated tumors, the salvage rate of eyes with miliary tumors was low (13\%). It is necessary to develop treatment schemes to effectively address miliary tumors and to minimize the development of them.

In a further study, the sub-analysis that divides patients into two groups, one with development of new tumor within cycles of chemotherapy and the other with development of new tumor after six cycles of chemotherapy, might be necessary to understand the comprehensive characteristics of new tumor development.

In summary, new small tumors develop in the forms of isolated or miliary tumors during and after chemotherapy. Younger children (less than 1 year at diagnosis) tend to develop isolated tumors during the follow-up period; whereas, patients with vitreal seeding are prone to be with miliary tumors which have less favorable salvage and visual outcomes. Stricter follow-up plans are required for patients who are diagnosed before the age of 1 year. Also, adequate management of vitreal seeding is essential in the treatment of retinoblastoma for the preservation of eyeball and vision.

\section{Conflict of Interest}

No potential conflict of interest relevant to this article was reported.

\section{References}

1. Shin JY, Kim JH, Yu YS, et al. Eye-preserving therapy in retinoblastoma: prolonged primary chemotherapy alone or combined with local therapy. Korean J Ophthalmol 2010;24:219-24.

2. Dimaras H, Corson TW, Cobrinik D, et al. Retinoblastoma. Nat Rev Dis Primers 2015;1:15021.

3. Abramson DH, Shields CL, Munier FL, Chantada GL. Treatment of retinoblastoma in 2015: agreement and disagreement. JAMA Ophthalmol 2015;133:1341-7.

4. Messmer EP, Sauerwein W, Heinrich T, et al. New and recurrent tumor foci following local treatment as well as ex- 
ternal beam radiation in eyes of patients with hereditary retinoblastoma. Graefes Arch Clin Exp Ophthalmol 1990;228:426-31.

5. Schueler AO, Anastassiou G, Jurklies C, et al. De novo intraocular retinoblastoma development after chemotherapy in patients with hereditary retinoblastoma. Retina 2006;26:425-31.

6. Shields CL, Shelil A, Cater J, et al. Development of new retinoblastomas after 6 cycles of chemoreduction for retinoblastoma in 162 eyes of 106 consecutive patients. Arch
Ophthalmol 2003;121:1571-6.

7. Murphree AL, Villablanca JG, Deegan WF 3rd, et al. Chemotherapy plus local treatment in the management of intraocular retinoblastoma. Arch Ophthalmol 1996;114:134856.

8. Kim H, Lee JW, Kang HJ, et al. Clinical results of chemotherapy based treatment in retinoblastoma patients: a single center experience. Cancer Res Treat 2008;40:164-71.

9. Abramson DH, Gombos DS. The topography of bilateral retinoblastoma lesions. Retina 1996;16:232-9. 